\title{
Chiral Surface Twists and Skyrmion Stability in Nanolayers of Cubic Helimagnets
}

\author{
A. O. Leonov, ${ }^{1,2,10,{ }^{*}}$ Y. Togawa, ${ }^{1,3,4,5}$ T. L. Monchesky, ${ }^{1,6}$ A. N. Bogdanov, ${ }^{1,2}$ J. Kishine, ${ }^{1,7}$ Y. Kousaka, ${ }^{1,8}$ \\ M. Miyagawa, ${ }^{1,8}$ T. Koyama, ${ }^{1,8}$ J. Akimitsu, ${ }^{1,8}$ Ts. Koyama, ${ }^{3}$ K. Harada, ${ }^{3}$ S. Mori, ${ }^{3}$ D. McGrouther, ${ }^{4}$ \\ R. Lamb, ${ }^{4}$ M. Krajnak, ${ }^{4}$ S. McVitie ${ }^{4}$ R. L. Stamps, ${ }^{4}$ and K. Inoue ${ }^{1,8,9}$ \\ ${ }^{1}$ Center for Chiral Science, Hiroshima University, Higashi-Hiroshima, Hiroshima 739-8526, Japan \\ ${ }^{2}$ IFW Dresden, Postfach 270016, D-01171 Dresden, Germany \\ ${ }^{3}$ Osaka Prefecture University, 1-2 Gakuencho, Sakai, Osaka 599-8570, Japan \\ ${ }^{4}$ School of Physics and Astronomy, University of Glasgow, Glasgow G12 8QQ, United Kingdom \\ ${ }_{5}^{5}$ JST, PRESTO, 4-1-8 Honcho Kawaguchi, Saitama 333-0012, Japan \\ ${ }^{6}$ Department of Physics and Atmospheric Science, Dalhousie University, Halifax, Nova Scotia B3H 3J5, Canada \\ ${ }^{7}$ The Open University of Japan, Chiba 261-8586, Japan \\ ${ }^{8}$ Graduate School of Science, Hiroshima University, Higashi-Hiroshima, Hiroshima 739-8526, Japan \\ ${ }^{9}$ IAMR, Facility of Science, Hiroshima University, Higashi-Hiroshima, Hiroshima 739-8530, Japan \\ ${ }^{10}$ Zernike Institute for Advanced Materials, University of Groningen, Nijenborgh 4, 9747 AG Groningen, Netherlands \\ (Received 16 January 2016; revised manuscript received 19 April 2016; published 15 August 2016)
}

\begin{abstract}
Theoretical analysis and Lorentz transmission electron microscopy (LTEM) investigations in an FeGe wedge demonstrate that chiral twists arising near the surfaces of noncentrosymmetric ferromagnets [Meynell et al., Phys. Rev. B 90, 014406 (2014)] provide a stabilization mechanism for magnetic Skyrmion lattices and helicoids in cubic helimagnet nanolayers. The magnetic phase diagram obtained for freestanding cubic helimagnet nanolayers shows that magnetization processes differ fundamentally from those in bulk cubic helimagnets and are characterized by the first-order transitions between modulated phases. LTEM investigations exhibit a series of hysteretic transformation processes among the modulated phases, which results in the formation of the multidomain patterns.
\end{abstract}

DOI: 10.1103/PhysRevLett.117.087202

Dzyaloshinskii-Moriya (DM) interactions [1] stabilize two-dimensional axisymmetric solitonic states (chiral Skyrmions) in saturated phases of magnetic materials with broken inversion symmetry [2,3]. Analytical and numerical studies reveal that, in two-dimensional uniaxial noncentrosymmetric ferromagnets, chiral magnetic Skyrmions condense into hexagonal lattices below a certain critical field and remain thermodynamically stable (they correspond to the global minimum of the magnetic energy functional) in a broad range of applied magnetic fields [3]. This does not occur in three-dimensional bulk cubic helimagnets where one-dimensional modulations along the applied field (the cone phase) [4] have the lowest energy in practically the whole area of the magnetic phase diagram, and Skyrmion lattices can exist only as metastable states $[5,6]$.

Recent observations of different types of magnetic Skyrmion states have been reported in freestanding nanolayers and epilayers of cubic helimagnets (e.g., Refs. [7-12]). These findings have given rise to a puzzling question: why are Skyrmion lattices suppressed in bulk cubic helimagnets but observed in nanolayers of the same material?

Published by the American Physical Society under the terms of the Creative Commons Attribution 3.0 License. Further distribution of this work must maintain attribution to the author(s) and the published article's title, journal citation, and DOI.
Two physical mechanisms have been proposed to date to explain the formation of Skyrmion lattices in nanolayers of cubic helimagnets. One of them is based on the effects imposed by induced uniaxial anisotropy [5,6]. In epilayers of cubic helimagnets on $\mathrm{Si}(111)$ substrates, a strong uniaxial anistropy is induced by the lattice mismatch between the B20 crystal and the substrate $[6,13]$. This uniaxial anisotropy suppresses the cone phase and stabilizes a number of nontrivial chiral modulated states including outof-plane and in-plane Skyrmion lattices recently observed in cubic helimagnet epilayers $[6,10,12]$.

The second stabilization mechanism is provided by specific modulations (chiral twists) arising near the surfaces of cubic helimagnet films [14-16]. Chiral twists have recently been discovered in $\mathrm{MnSi} / \mathrm{Si}(111)$ films $[14,16]$. However, their influence on the magnetic states arising in the freestanding films of cubic helimagnets is still unclear. Also, physical mechanisms underlying the formation of Skyrmionic states in such films are unknown, and a theoretical description of the magnetic states in these systems is still an open question.

In this Letter we report on a theoretical analysis of magnetic modulated states with confined magnetic structures at the boundaries in cubic helimagnets, which we term confined cubic helimagnets, and our findings show that surface twist instabilities play a decisive role in the stabilization of 


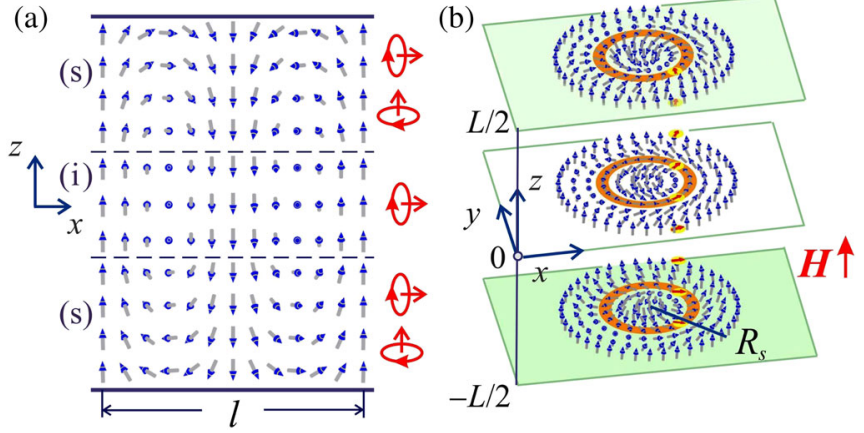

FIG. 1. Magnetic structure of (a) a helicoid with period $l$ and (b) a Skyrmion lattice cell of radius $R_{s}$, in nanolayers of cubic helimagnets. In the internal area (i), the helicoid has in-plane modulations along the $x$ axis, the surface areas (s) are modulated along the $x$ and $z$ axes (arrows with circles indicate the rotation sense and propagation directions).

Skyrmionic states and give rise to the first-order transitions between modulated phases in freestanding layers of cubic helimagnets. A series of images in an FeGe wedge specimen obtained by using Lorentz transmission electron microscopy (LTEM) revealed hysteretic transformations and the coexistence of modulated states, which are supportive of theoretical calculations.

The standard model for magnetic states in cubic noncentrosymmetric ferromagnets is based on the energy density functional $[1,4]$

$$
w=A(\operatorname{grad} \mathbf{m})^{2}+D \mathbf{m} \cdot \operatorname{rot} \mathbf{m}-\mu_{0} M \mathbf{m} \cdot \mathbf{H},
$$

including the principal interactions essential to stabilizing modulated states: the exchange stiffness with constant $A$, DM coupling energy with constant $D$, and the Zeeman energy; $\mathbf{m}=(\sin \theta \cos \psi ; \sin \theta \sin \psi ; \cos \theta)$ is the unity vector along the magnetization vector $\mathbf{M}=\mathbf{m} M$, and $\mathbf{H}$ is the applied magnetic field.

We investigate the functional (1) in a film of thickness $L$ infinite in the $x$ and $y$ directions and confined by parallel planes at $z= \pm L / 2$ in magnetic field $\mathbf{H}$ applied along the $z$ axis [Fig. 1(a)]. The equilibrium magnetic states in the film are derived by the Euler equations for energy functional (1) together with the Maxwell equations and with corresponding boundary conditions. The solutions depend on the two control parameters of the model (1), the confinement ratio, $\nu=L / L_{D}$, and the reduced value of the applied magnetic field, $h=H / H_{D}$, where $L_{D}=4 \pi A /|D|$ is the helix period and $\mu_{0} H_{D}=D^{2} /(2 A M)$ is the saturation field $[3,4]$.

The solutions for unconfined helicoids [1] and Skyrmion lattices [3] homogeneous along the film normal (the $z$ axis) describe magnetic configurations in the depth of a bulk cubic helimagnet. However, the situation changes radically near the film surfaces. The gradient term,

$$
m_{x} \partial m_{y} / \partial z-m_{y} \partial m_{x} / \partial z
$$

in the DM energy functional [Eq. (1)] violates transversal homogeneity of helicoids and Skyrmion states and imposes chiral modulations along the $z$ axis that decay into the depth of the sample (surface twists) $[6,14,15]$. The penetration depth of these surface modulations is estimated as 0.1 $L_{D}[6]$.

Mathematically, axisymmetric Skyrmion cells in thin films are described by solutions of type $\theta=\theta(\rho, z)$, $\psi=\psi(\varphi, z)$, and helicoids propagating in a film along the $x$ axis are described by solutions of type $\theta(x, z)$, $\psi(x, z)$. The equilibrium solutions for confined helicoids and Skyrmion lattices are derived by solving the Euler equations for functional (1) with free boundary conditions at the film surfaces $(z= \pm L / 2)$.

Most of the investigated freestanding films and epilayers of cubic helimagnets have a thickness exceeding the period of the helix $\left(L \geq L_{D}\right)$ [8-12]. Therefore, in this Letter we carry out a detailed analysis of the solutions for confined chiral modulations in cubic helimagnetic films with the thickness ranging from $L=L_{D}$ to a bulk limit $\left(L \gg L_{D}\right)$.

The calculated $\nu-h$ phase diagram in Fig. 2 indicates the areas with the chiral modulated states corresponding to the global minimum of the energy functional and separated by the first-order transition lines. For $L \gg L_{D}$ the solutions for confined helicoids and Skyrmion lattices approach the solutions for the magnetic states in the unconfined case, which are homogeneous along the $z$ axis $[1,3]$. Surface twist instabilities arising in confined cubic helimagnets $[15,16]$ provide a thermodynamical stability for helicoids and Skyrmion lattices in a broad range of the applied fields (Fig. 2).

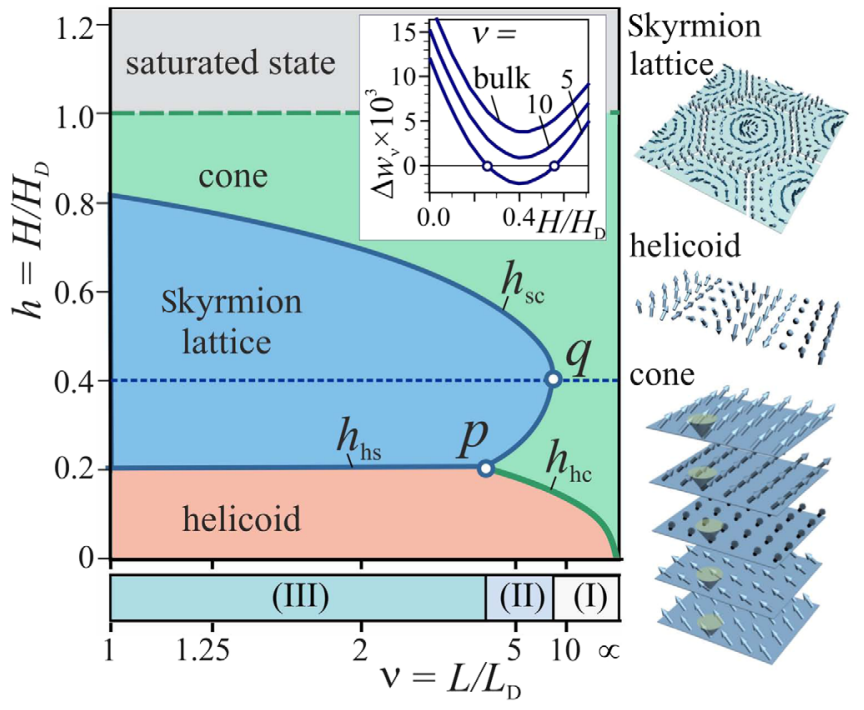

FIG. 2. The magnetic phase diagram of the magnetic states corresponding to the global minima for model (1) in reduced variables for the film thickness $\nu=L / L_{D}$ and the applied magnetic field $h=H / H_{D}$. The existence areas of the modulated phases (cone, helicoids, and Skyrmion lattice) are separated by the first-order transition lines (solid). $p(4.47,0.232)$ is a triple point, $q(7.56,0.40)$ is a completion point. A dashed line indicates the second-order transition between the cone and the saturated state. Along the dotted line $H_{a}=0.4 H_{D}$, the difference between the energy densities of the Skyrmion lattice and the cone phase $\left(\Delta w_{\nu}\right)$ is minimal (see the inset). 
Another noticeable feature of the phase diagram is that the line $h=0.4$ is a symmetry axis for the Skyrmion lattice stability area [6]. This follows from the fact that, in bulk helimagnets, this field corresponds to the minimal value of the Skyrmion lattice energy compared to that of the cone phase $[5,6]$. The differences between the equilibrium average energy densities of the Skyrmion lattice $\left(\bar{w}_{\mathrm{s}}\right)$ and the energy density of the cone phase $\left(w_{\mathrm{c}}\right) \Delta w_{\nu}(h)=$ $\bar{w}_{\mathrm{s}}(h, \nu)-w_{\mathrm{c}}(h, \nu)$ plotted as functions of the applied field also reach the minimum in the fields close to $h=0.4$ (inset of Fig. 2). As a result, below $\nu_{q}=7.56$, the stability area of the Skyrmion lattices extends around the line $h=0.4$. Near the ordering temperature, $\Delta w_{\nu}(h)$ becomes anomalously small, and even such weak interactions as cubic anisotropy can stabilize Skyrmion lattices in this area. This effect plays a crucial role in the formation of the $A$-phase pocket near the ordering temperature of the bulk cubic helimagnets (for details, see Refs. $[6,17]$ ).

In the whole range of the film thickness, the helicoids with in-plane propagation directions correspond to the ground state of the system. The triple point $p(4.47,0.232)$ and the completion point $q(7.56,0.40)$ split the phase diagram into three distinct areas with different types of magnetization processes. (1) $\nu>\nu_{q}=7.56$. In these comparatively thick films, the helicoids remain thermodynamically stable at low fields and transform into the cone by a first-order process at the critical line $h_{\mathrm{hc}}(\nu)$. The cone magnetization along the applied field increases linearly for an increasing magnetic field up to the saturation at critical field $h=H / H_{D}=1$. (2) $4.47=\nu_{p}<\nu<\nu_{q}=7.56$. In this case, the magnetic-field-driven evolution of the cone is interrupted by the first-order transition in the Skyrmion lattice at $h_{\mathrm{hc}}(\nu)<h_{q}$ and the reentrant transition at $h_{\mathrm{hc}}(\nu)>h_{q}$. (3) $1<\nu<\nu_{p}=4.47$. In this thickness range, the stability area of Skyrmion lattices is separated from the low field helicoid and high field cone phases by the first-order transition lines.

The magnetic phase diagram in Fig. 2 has been derived by a minimization of the simplified ("isotropic") energy functional (1) with free boundary conditions. This demonstrates how a pure geometrical factor (confinement) influences the energetics of cubic helimagnet nanolayers by imposing transverse chiral modulations (twists) in Skyrmion lattices and helicoids. This phase diagram is representative for a manifold of cubic helimagnet freestanding layers with different types of surface twists. This describes a general topology of the $(\nu, h)$ phase diagrams only. Depending on the parameters of the localized surface twists, the values of critical points and location of the critical lines in the $(\nu, h)$ diagrams can differ strongly from those in Fig. 2.

In a recently published paper [18], the authors demonstrate that surface modulations propagating perpendicularly to the film surfaces arise in the cone phase below a magnetic field $h=0.42$. Importantly, these surface perturbations are localized near the film surfaces and do not influence the main part of the film volume. According to classical thermodynamics, this spin texture should be identified as the cone phase. Similarly, the helicoids and Skyrmion lattices with localized surface twists should be designated as the helicoid and Skyrmion lattice phases (Fig. 2).

Iron monogermanide (FeGe) belongs to a group of noncentrosymmetric cubic helimagnets (with space group $P 2_{1} 3$, B20-type structure) [17,19]. Below the Curie temperature $T_{C}=278.2 \mathrm{~K}, \mathrm{FeGe}$ is ordered into homochiral helices with period $L_{D}=70 \mathrm{~nm}$ propagating along equivalent $\langle 100\rangle$ directions [19]. Below $T_{1}=211 \mathrm{~K}$, helices propagate along the $\langle 111\rangle$ directions. For increasing temperature, the propagation directions $\langle 100\rangle$ are restored at $T_{2}=245 \mathrm{~K}$ [19]. In bulk cubic helimagnets, onedimensional single-harmonic chiral modulations (helices and cones) are observed as stable states over practically the entire region below the saturation field [19]. In contrast to bulk specimens, in freestanding nanolayers of cubic helimagnets with a thickness $L \leq 120 \mathrm{~nm}$ investigated by LTEM methods, Skyrmion lattices and helicoids are observed in broad ranges of applied magnetic fields and temperatures, while the cone phase is partially or completely suppressed [7-9]. Recent LTEM investigations represent an extensive study of the evolution of Skyrmion states in confined cubic helimagnets (see, e.g., Refs. [7-9,20] and the bibliography in Ref. [21]).

In our Letter we use LTEM to explore the experimental evidence of the first-order phase transitions into the cone phase and other specific magnetization processes imposed by the chiral surface twists (Fig. 2). For our studies, we have prepared wedge-shaped single crystal FeGe(110) films. FeGe single crystals were grown by a chemical vapor transport method. A thin film specimen was made for TEM observations by using a focused ion beam technique.

A series of Lorentz micrographs were taken by means of a Fresnel mode of Lorentz microscopy [22,23] with a typical defocus value of $10 \mu \mathrm{m}$ at $T=110$ and $250 \mathrm{~K}$ in a broad range of magnetic fields applied perpendicular to the film surface (Figs. 3 and 4). Fresnel imaging reveals contrast at positions where there is variation of the inplane component of the magnetic induction, which can be interpreted as the magnetization in this case. Such images clearly reveal the magnetic-field-driven first-order transitions between the basic modulated states (helicoids and Skyrmions appearing as bright and/or dark stripes or spots, respectively) accompanied by the formation of the multidomain patterns composed of domains of the competing phases. It should be noted that, for transitions to the conical state, there is no distinctive contrast which unambiguously identifies this phase. Where we identify such transitions to the conical state, we are aware that the lack of contrast could also be consistent with other phases such as saturated, paramagnetic, and nonmagnetic states. However, in Figs. 3 and 4 these regions exist at applied fields lower 

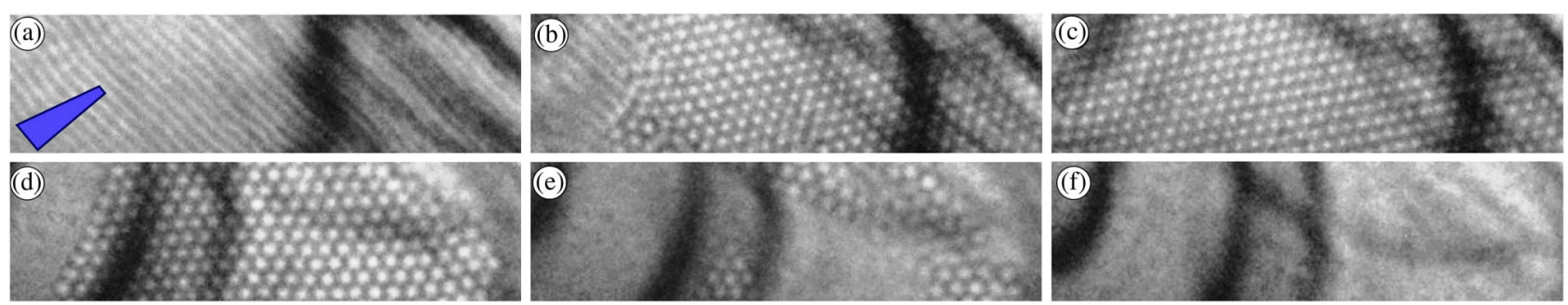

FIG. 3. LTEM images of modulated phases in an FeGe wedge at $T=250 \mathrm{~K}$ and different values of the applied field $H$ (T): (a) 0.013 , (b) 0.0873 , (c) 0.1073 , (d) 0.2215 , (e) 0.2355 , (f) 0.3728 . The defocus value is $10 \mu \mathrm{m}$. (c) indicates the coexisting helicoid and Skyrmion lattice states and (d),(e) the Skyrmion lattice and cone domains during the first-order phase transitions. The image size is $3000 \mathrm{~nm} \times 800 \mathrm{~nm}$, the thickness varies from $120 \mathrm{~nm}$ (left) to $60 \mathrm{~nm}$ (right). Blue tetragons indicate the direction of the thickness gradients (in this and the following figures).

than the saturated fields (for FeGe, the saturation field 0 $\mathrm{HD}=0.359 \mathrm{~T}[17,19])$. Moreover, according to the theoretical results [1,3] and experimental observations [7,22], the magnetic-field-driven transitions of the helicoid and the Skyrmion lattice into the saturated state advance gradually by the extension of the modulation period and the formation of isolated helicoidal kinks and Skyrmions. These processes should exclude the formation of multidomain patterns of the competing phases characteristic of the first-order transitions [24].

In Fig. 3, the layer thickness varies from $L=120 \mathrm{~nm}$ $(\nu=1.7)$ at the left edge to $L=60 \mathrm{~nm}(\nu=0.86)$ at the right edge. In the calculated phase diagram, this thickness interval $(0.86<\nu<1.7)$ belongs to area III, shown in Fig. 2, characterized by the first-order transitions between the helicoid and the Skyrmion lattice at the lower field, $h_{h s}(\nu)$, and between the Skyrmion lattice and the cone at the higher field, $h_{s c}(\nu)$ (Fig. 2). Both of these phase transitions are clearly observed in Fig. 3. We stress that because the transition field $h_{s c}(\nu)$ has lower values for larger $\nu$, initially the cone phase nucleates at the thicker edge of the film (Figs. 3 and 4) and expands to the thinner part with an increasing applied field (Figs. 3 and 4).

The LTEM images taken at $T=110 \mathrm{~K}$ (Fig. 4) correspond to a wedge area belonging to the same thickness interval as that in Fig. 3, with a thickness variation ranging from $L=90 \mathrm{~nm}(\nu=1.29)$ at the bottom edge to $L=$ $60 \mathrm{~nm}(\nu=0.86)$ at the top edge. However, the magnetization evolution differs drastically from that observed at higher temperature. In this case, the Skyrmion lattice does not arise; instead, the helicoid directly transforms into the cone phase at a considerably lower field of about $0.1 \mathrm{~T}$ by a first-order process [Figs. 4(b) and 4(c)]. In the $(\nu, h)$ phase diagram (Fig. 2), such a magnetization evolution occurs in area I for $\nu>\nu_{q}=7.56$. The suppression of Skyrmion lattices and helicoids at lower temperatures is characteristic for freestanding cubic helimagnet nanolayers [8,9]. Particularly, at $T=110 \mathrm{~K}$, the Skyrmion lattices arise in FeGe freestanding layers only when their thickness is smaller than $35 \mathrm{~nm}$ [8]. This effect can be understood if we assume that the surface energy imposed by chiral twists decreases with decreasing temperatures. As a result, at lower temperatures the existence area of Skyrmion lattices in the $(\nu, h)$ phase diagram (2) would be shifted into the region with a lower $\nu$.

The results of micromagnetic calculations for confined chiral modulations demonstrate that chiral surface twists provide the stabilization mechanism for helicoids and Skyrmion lattices in freestanding cubic helimagnet films [25]. The solutions minimizing the energy functional (1) with free boundary conditions describe chiral modulations imposed solely by the geometrical confinement and expose three basic types of magnetization processes in cubic helimagnet nanolayers (Fig. 2). LTEM investigations of magnetic states in an FeGe wedge specimen reveals hysteretic formation and the coexistence of modulated phases far beyond the equilibrium regime of the phase diagram, which is consistent with the first-order nature of the phase transition theoretically predicted. In a real system, the confined chiral modulations may arise as a result of the
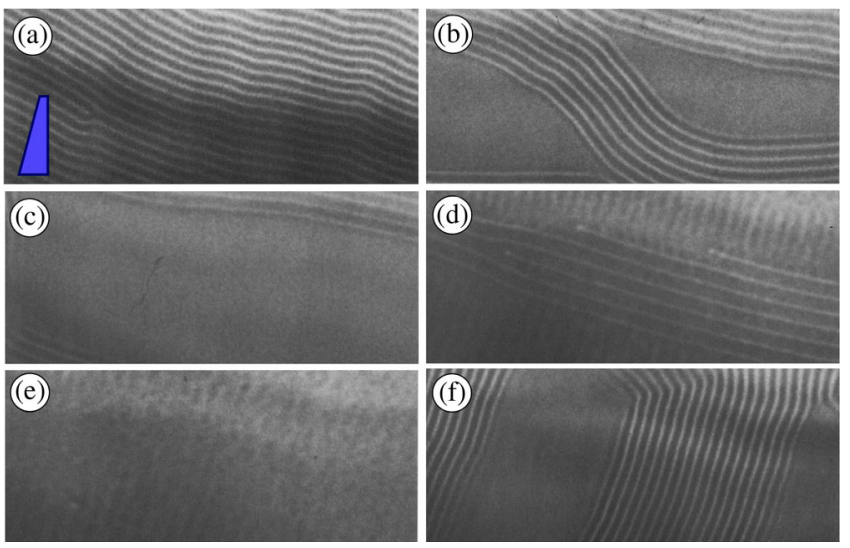

FIG. 4. LTEM images of an FeGe wedge at $T=110 \mathrm{~K}$ for applied magnetic fields: (a) $H$ (T) $=0.02$, (b) 0.1074 , (c) 0.146 , (d) 0.32 , (e) 0.367 , (f) 0.02 . The defocus value is $2 \mu \mathrm{m}$. (b),(c) Coexisting domains of the helicoid and cone state during the firstorder transition between these phases. Multidomain states are restored after decreasing the applied field (f). The image size is $3000 \mathrm{~nm} \times 1250 \mathrm{~nm}$, the thickness varies from $90 \mathrm{~nm}$ (bottom) to $60 \mathrm{~nm}$ (top). 
interplay between the stabilization mechanism imposed by the geometrical confinement and other physical factors, such as intrinsic cubic anisotropy and induced volume and surface uniaxial anisotropy, and internal and surface demagnetization effects. Our findings provide a conceptional basis for detailed experimental and theoretical investigations of the complex physical processes underlying the formation of Skyrmion lattices and helicoids in confined magnets.

In addition, the open access data link is provided in Ref. [26].

The authors are grateful to H. Fukuyama and G. Tatara for the useful discussions. We acknowledge support from the JSPS Grant-in-Aid for ScientificResearch (S) (Grant No. 25220803), JSPS Core-to-Core Program A, Advanced Research Networks, the MEXT program for promoting the enhancement of research universities (Hiroshima University), and Grant from EPSRC No. EP/M024423/1. A. O. L acknowledges financial support from FOM Grant No. 11PR2928. A. N. B acknowledges support from the Deutsche Forschungsgemeinschaft via Grant No. BO 4160/ 1-1. Y. T. acknowledges financial support from JSPS Brain Circulation Project (R2507).

*A.Leonov@ifw-dresden.de

[1] I. E. Dzyaloshinskii, Sov. Phys. JETP 19, 960 (1964); 20, 665 (1965).

[2] A. N. Bogdanov and D. A. Yablonskii, Zh. Eksp. Teor. Fiz. 95, 178 (1989) [Sov. Phys. JETP 68, 101 (1989)].

[3] A. Bogdanov and A. Hubert, J. Magn. Magn. Mater. 138, 255 (1994); 195, 182 (1999).

[4] P. Bak and M. H. Jensen, J. Phys. C 13, L881 (1980).

[5] A. B. Butenko, A. A. Leonov, U. K. Rößler, and A. N. Bogdanov, Phys. Rev. B 82, 052403 (2010).

[6] M. N. Wilson, A. B. Butenko, A. N. Bogdanov, and T. L. Monchesky, Phys. Rev. B 89, 094411 (2014).

[7] X. Z. Yu, Y. Onose, N. Kanazawa, J. H. Park, J. H. Han, Y. Matsui, N. Nagaosa, and Y. Tokura, Nature (London) 465, 901 (2010).

[8] X. Z. Yu, N. Kanazawa, Y. Onose, K. Kimoto, W. Z. Zhang, S. Ishiwata, Y. Matsui, and Y. Tokura, Nat. Mater. 10, 106 (2011).

[9] X. Z. Yu, A. Kikkawa, D. Morikawa, K. Shibata, Y. Tokunaga, Y. Taguchi, and Y. Tokura, Phys. Rev. B 91, 054411 (2015).
[10] M. N. Wilson, E. A. Karhu, A. S. Quigley, U. K. Rößler, A. B. Butenko, A. N. Bogdanov, M. D. Robertson, and T. L. Monchesky, Phys. Rev. B 86, 144420 (2012).

[11] T. Yokouchi, N. Kanazawa, A. Tsukazaki, Y. Kozuka, A. Kikkawa, Y. Taguchi, M. Kawasaki, M. Ichikawa, F. Kagawa, and Y. Tokura, J. Phys. Soc. Jpn. 84, 104708 (2015)

[12] S. X. Huang and C. L. Chien, Phys. Rev. Lett. 108, 267201 (2012).

[13] E. Karhu, S. Kahwaji, T. L. Monchesky, C. Parsons, M. D. Robertson, and C. Maunders, Phys. Rev. B 82, 184417 (2010); E. A. Karhu, S. Kahwaji, M. D. Robertson, H. Fritzsche, B. J. Kirby, C. F. Majkrzak, and T. L. Monchesky, Phys. Rev. B 84, 060404 (2011).

[14] M. N. Wilson, E. A. Karhu, D. P. Lake, A. S. Quigley, S. Meynell, A. N. Bogdanov, H. Fritzsche, U. K. Rößler, and T. L. Monchesky, Phys. Rev. B 88, 214420 (2013).

[15] F. N. Rybakov, A. B. Borisov, and A. N. Bogdanov, Phys. Rev. B 87, 094424 (2013); F. N. Rybakov, A. B. Borisov, S. Blügel, and N. S. Kiselev, Phys. Rev. Lett. 115, 117201 (2015).

[16] S. A. Meynell, M. N. Wilson, H. Fritzsche, A. N. Bogdanov, and T. L. Monchesky, Phys. Rev. B 90, 014406 (2014).

[17] H. Wilhelm, M. Baenitz, M. Schmidt, C. Naylor, R. Lortz, U. K. Rößler, A. A. Leonov, and A. N. Bogdanov, J. Phys. Condens. Matter 24, 294204 (2012).

[18] F. N. Rybakov, A. B. Borisov, S. Blügel, and N. S. Kiselev, New J. Phys. 18, 045002 (2016).

[19] B. Lebech, J. Bernhard, and T. Freltoft, J. Phys. Condens. Matter 1, 6105 (1989).

[20] Y. Onose, Y. Okamura, S. Seki, S. Ishiwata, and Y. Tokura, Phys. Rev. Lett. 109, 037603 (2012).

[21] N. Nagaosa and Y. Tokura, Nat. Nanotechnol. 8, 899 (2013).

[22] Y. Togawa, T. Koyama, K. Takayanagi, S. Mori, Y. Kousaka, J. Akimitsu, S. Nishihara, K. Inoue, A. S. Ovchinnikov, and J. I. Kishine, Phys. Rev. Lett. 108, 107202 (2012).

[23] S. McVitie and M. Cushley, Ultramicroscopy 106, 423 (2006).

[24] A. Hubert and R. Schäfer, Magnetic Domains (Springer, Berlin, 1998); V. G. Baryakhtar, A. N. Bogdanov, and D. A. Yablonskii, Sov. Phys. Usp. 31, 810 (1988).

[25] See Supplemental Material at http://link.aps.org/ supplemental/10.1103/PhysRevLett.117.087202 for details of analytical solutions for chiral surface twists and details of thin film preparation of FeGe helimagnet.

[26] DOI: 10.5525/gla.researchdata.328. 